\title{
AVALIAÇÃO DA SENSIBILIDADE DA TÉCNICA DE ECLOSÃO DE MIRACÍDIOS, DE SUZUKI, EM COMPARAÇÃO COM OUTROS PROCESSOS EMPREGADOS NO DIAGNÓSTICO DA ESQUISTOSSOMOSE MANSÔNICA
}

\author{
Pedro Luiz Silva Pinto, Rubens Campos, Vicente \\ Amato Neto, Antônio Augusto Baillot Moreira, \\ Eunice José de Sant'Ana, Elaine Guizelini' ${ }^{1}$ e \\ Naim Sauaia.
}

\begin{abstract}
Para simplificar o diagnóstico da esquistossomose mansônica há interesse de utilizar exame das fezes executado com base na eclosão de miracidios. Foi analisada a sensibilidade do método idealizado por Suzuki, através de comparação com os processos de Kato-Katz, Hoffman, Pons e Janer (sedimentação espontânea em água) e Chieffi e cols. Ficou comprovado que a tática em apreço é útil, mas não suplanta os méritos das outras empregadas, apresentando todavia melhor resultado quando presentes nas fezes maiores quantidades de ovos.
\end{abstract}

Palavras chaves: Esquistossomose mansônica. Diagnóstico. Eclosão de miracídios. Técnica de Suzuki.

Para estabelecer o diagnóstico da esquistossomose mansônica, o adequado exame parasitológico das fezes corresponde, sem dúvida, ao procedimento mais comumente usado, contando com a vantagem de propiciar, através da evidenciação de ovos caracteristicos, categórica especificidade. Prova intradérmica e métodos sorológicos são também úteis a respeito, mas dependem de materiais e equipamentos não baratos, convindo frisar que é judicioso considerá-los aproveitáveis sobretudo para a realização de inquéritos epidemiológicos.

Diversas técnicas afiguram-se adequadas para a evidenciação de ovos de Schistosoma mansoni na matéria fecal. Elas possuem propriedades diferentes e, sob o ponto de vista técnico, têm nexo com execuções que envolvem simplicidades de graus diversos. Algumas dessas técnicas incluem, de fato, sofisticação um pouco maior, mas de qualquer forma todas elas não requerem atributos ou recursos de porte especial.

No contexto pertinente à análise das fezes, têm lugar os processos que se baseiam na visualização de miracidios, apregoados porque podem permitir maiores facilidades e rendimento operacional, com dispensa de instrumentos custosos e especiais 2456891011 . Como fruto de nosso constante interesse no que tange ao diagnóstico de parasitoses intestinais, tomamos conhecimento de técnica proposta por Suzuki ${ }^{10}$ e calcada na

Trabalho do Laboratório de Investigação Médica - Parasitologia, do Hospital das Clínicas, da Faculdade de Medicina da Universidade de São Paulo, São Paulo, SP

Recebido para publicaçào em 17/10/84 demonstração dos já mencionados miracidios. Consideramos, então, conveniente demarcar a utilidade dela, por meio do estudo agora relatado.

\section{MATERIAL E MÉTODOS}

Nossas verificações dizem respeito a dois grupos (A e B), estipulados de acordo com a procedencia das fezes examinadas: A) 103 amostras, de indivíduos certamente infectados pelo $S$. mansoni, triadas pelas técnicas de Kato-Katz e de Hoffman, Pons e Janer, rotineiramente utilizadas na Seção de Parasitologia do Laboratório Central, do Hospital das Clínicas, da Faculdade de Medicina da Universidade de São Paulo; B) 100 amostras enviadas ao laboratório para caracterização de esquistossomose mansônica, existindo, em virtude de motivos de várias ordens, suspeita da presença dessa helmintiase.

Analisamos todos esses materiais por meio de quatro técnicas: de sedimentação espontânea em água (Hoffman, Pons e Janer), empregando 2,0 g de fezes e observando o materiál contido entre làmina e lamínula ( $22 \times 22 \mathrm{~mm}$ ); de Kato-Katz, quantitativa; de eclosão de miracidios, segundo Chieffi e cols ${ }^{2}$; de eclosão de miracidios, segundo Suzuki ${ }^{10}$. As formas de agir correspondentes às duas primeiras estão descritas no compêndio especializado de autoria de Amato Neto e Corrêa ${ }^{1}$; $a$ atinente à terceira encontra-se especificada na publicação indicada e, a seguir, esmiuçamos a idealizada por Suzuki, por ser ela muito pouco divulgada no Brasil: emulsionar cerca de 2,0 $\mathrm{g}$ de fezes em solução de cloreto de sodio a $0,85 \%$; coar em gaze (quatro 
Pinto PLS, Campos R, Amato Neto V, Moreira AAB, Sant'Ana EJ, Guizelini E, Sauaia N. Avaliacão da sensibilidade da técnica de eclosão de miracidios, de Suzuki, em comparação com outros processos empregados no diagnóstico da esquistossomose mansônica. Revista da Sociedade Brasileira de Medicina Tropical 19: 85-88, Abr-Jun, 1986

dobras); deixar sedimentar durante 30 minutos; lavar o sedimento, com a solução citada, desprezando o sobrenadante e ressuspendendo a cada 15 minutos, para obter liquido limpido; filtrar a suspensão através de papel de filtro, em funil de Büchner conectado com frasco de Kitasato ligado a trompa de vácuo; aspirar até que o fluido desapareça no papel de filtro; cortar o papel que contém o sedimento e colocar em Erlenmeyer de $125 \mathrm{ml}$, contendo água declorada; manter sob foco de luz, constituido por lâmpada de 60 watts; observar, a olho nu, a superficie do frasco, realizando cerca de cinco apreciaçōes, intervaladas por 15 a 30 minutos, a fim de verificar a presença ou não de miracidios (Fig. 1).

Os resultados registrados para os quatro métodos, em ambos os grupos, sofreram processamento estatístico por meio do teste de Cochran. A avaliação da discordância entre o método de Kato-Katz, tomado como referência e o de Suzuki, assim como também entre os de eclosão entre si, foi feita pelo teste de McNemar, adotando-se nivel de significância de 0,05 37 .

\section{RESULTADOS}

Nossas verificações fundamentais estão consignadas nas Tabelas 1 e 2 . $\mathrm{Na}$ primeira, figuram as positividades em termos absolutos e respectivos percentuais para cada uma das quatro técnicas e, na segunda, determinaçōes quantitativas indicadas pela de KatoKatz.
O limite adotado de 72 ovos por grama de fezes para efeito de agrupamento das amostras, foi baseado na simples observação empirica da diferença de comportamento entre os métodos a partir desse nível, havendo a seguir apreciação por testes estatísticos.

$O$ valor da estatística de Cochran, quando aplicado no grupo $A$ correspondeu a $Q=4,385$ (valor crítico 7,815), o que evidencia não existir diferença significante entre os quatro processos. No entanto, quando aplicado no grupo $B$, obtivemos $Q=9,834$, indicando existir desigualdades entre as referidas técnicas, neste grupo.

No confronto, especificamente, dos métodos de Kato-Katz e Suzuki, a discordância mostrou-se significante em ambos os grupos, fornecendo o primeiro maior frequeencia de resultados positivos (teste de McNemar).

Quando comparadas as técnicas de eclosão (Suzuki e Chieffi e cols), no grupo A, para amostras agrupadas com número menor ou igual a 72 ovos por grama de fezes, a divergência foi estatisticamente significante, tendo a técnica de Chieffi e cols, neste caso, revelado maior número de exames positivos. Contudo, quando comparadas com as amostras com cifra maior do que 72, as duas não se apresentaram diversas. Para o grupo B, não houve discordância tanto entre os materiais agrupados até 72 ovos por grama de fezes, como acima desse valor.

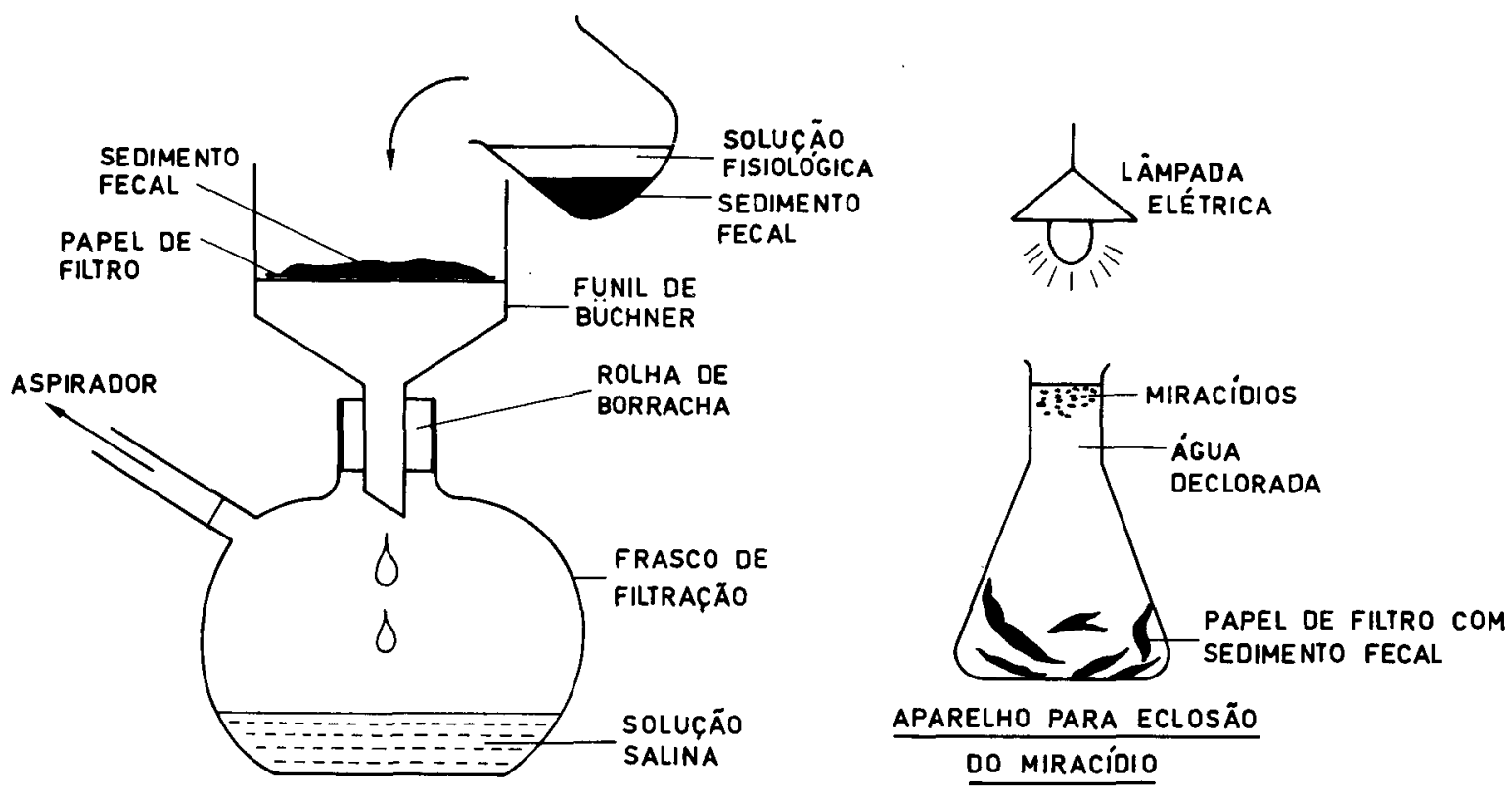

APARELHO PARA LAVAGEM

DO SEDIMENTO FECAL COM SOLUÇÄO FISIOLÓGICA

Fig. 1 - Etapas e equipamentos referentes à execução do método de Suzuki, para eclosão de miracidios. 
Pinto PLS, Campos R, Amato Neto V, Moreira AAB, Sant'Ana EJ, Guizelini E, Sauaia N. Avaliação da sensibilidade da técnica de eclosão de miracídios, de Suzuki, em comparação com outros processos empregados no diagnóstico da esquistossomose mansônica. Revista da Sociedade Brasileira de Medicina Tropical 19: 85-88, Abr-Jun, 1986

Tabela 1 - Números de resultados positivos pelas quatro técnicas, segundo os grupos $A$ e $B$.

\begin{tabular}{lcccc}
\hline & \multicolumn{4}{c}{ Técnica } \\
\cline { 2 - 5 } Grupo & Kato-Katz & $\begin{array}{c}\text { Hoffman, Pons } \\
\text { e Janer }\end{array}$ & Chieffi e cols. & Suzuki \\
\hline A (103 casos) & $95(92,2 \%)$ & $85(82,5 \%)$ & $90(87,3 \%)$ & $80(77,6 \%)$ \\
B (37 casos) & $32(86,4 \%)$ & $25(67,5 \%)$ & $23(62,1 \%)$ & $24(64,8 \%)$ \\
\hline
\end{tabular}

Tabela 2 - Positividades pelas quatro técnicas, segundo os grupos $A$ e $B$, ao ser levado em conta critério quantitativo (Kato-Katz).

\begin{tabular}{lcccc}
\hline \multirow{2}{*}{$\begin{array}{c}\text { Ovos por grama } \\
\text { de fezes }\end{array}$} & Grupo & Kato-Katz & Chieffi e cols. & Suzuki \\
\cline { 3 - 5 } Até 72 & A & 25 & 29 & 19 \\
& B & 15 & 7 & 8 \\
\hline \multirow{2}{*}{ Mais do que 72} & A & 70 & 61 & 61 \\
& B & 17 & 16 & 16 \\
\hline
\end{tabular}

\section{DISCUSSÃO}

Os dados que coletamos permitiram a mensuração da sensibilidade da técnica de eclosão de miracidios preconizada por Suzuki. A observação dos dados constantes da Tabela 1, onde anotamos os percentuais de positividade de $77,6 \%$ e $64,8 \%$, respectivamente para um e outro grupo de casus estudados e obtidos pela aplicação do método, comprova ser ele razoavelmente eficiente, a despeito de deixar de revelar a parasitose em número não desprezivel de situações.

O processamento estatístico instituido para amparar nossos resultados, com aplicação de dois testes de comparação (Cochran e McNemar) teve sempre fundamento na colocação da técnica de KatoKatz como referencial. Isto pela possibilidade de permitir a extração de dados com base no conhecimento da carga parasitária, como também por ser ela a que, desde o inicio, revelou o maior número de positividades.

$O$ intuito preliminar da inclusão de dois grupos no estudo, qual seja o de minimizar a interferência de possiveis fatores de ordem subjetiva na execução dos processos e interpretação dos resultados, propiciou por vezes conclusões de ordem estatística aparentemente contraditórias. Isto, cremos, não invalida as conclusões finais, convindo atentar para aspectos de ordens biológica e epidemiológica importantes, como a possibilidade da casuistica de um ou do outro grupo incluir indivíduos de procedências diversas, em que pese a reconhecida maior sensibilidade dos métodos de eclosão em amostras provindas de área endêmica. Além do fato de estarmos confrontando dados oriundos de fenómenos distintos, como presença de ovos nos procedimentos de Kato-Katz e Hoffman, Pons e Janer e de miracidios nos de eclosão ${ }^{2}$.

Nenhum processo é infalivel ao ser aplicado para diagnosticar a esquistossomose e, à comparação que levamos a efeito, o de Kato-Katz despontou como melhor, apesar do comentário anteriormente emitido.

Quando valorizada a intensidade da parasitose, que sugeria, de modo empirico, comportamentos dispares segundo a carga parasitária, com evidenciação de aumento da sensibilidade nas infecções mais relevantes, consideradas ambas as técnicas de eclosão, não se conseguiu, todavia, diferenciá-la integralmente. Isto, por falta de respaldo no processamento estatístico efetuado, pois somente para o grupo A e amostras com numero menor ou igual a 72 ovos por grama de fezes, houve discordância significante, denotando uma melhor performance da técnica de Chieffi e cols.

É lícito reconhecer que o método de Suzuki não prima por encerrar a simplicidade ansiada, quanto à 
Pinto PLS, Campos R, Amato Neto V, Moreira AAB, Sant'Ana EJ, Guizelini E, Sauaia N. Avaliação da sensibilidade da técnica de eclosão de miracidios, de Suzuki, em comparação com outros processos empregados no diagnóstico da esquistossomose mansônica. Revista da Sociedade Brasileira de Medicina Tropical 19: 85-88, Abr-Jun, 1986

execução. De qualquer forma, trata-se de mais uma conduta fundamentada na eclosão de miracidios, presentemente alvo das especulaçōes que comunicamos. Ela e outras congêneres, se preferidas em trabalhos vinculados à esquistossomose mansônica, devem estar suficientemente amparadas em pesquisas que procuraram mensurar suas validades. Talvez, então até possam merecer empregos, após correções por meio de coeficientes.

\section{SUMMARY}

In order to simplify the diagnosis of Manson's schistosomiasis, the use of stool examinations based miracidial hatching has recently been considered. The sensitivity of the Suzuki's technique was evaluated in comparison to those of Kato-Katz, Hoffman, Pons and Janer and Chieff and cols. The former technique showed itself to be useful, yielding better results when large numbers of eggs were found in the stool, but did not surpass the performance of the other above mentioned techniques.

Key' words: Manson's schistosomiasis. Diagnosis. Miracidium hatching. Suzuki's technique.

\section{REFERÊNCIAS BIBLIOGRÁFICAS}

1. Amato Neto V. Corrèa LL. Exame parasitologico das fezes. Sarvier, São Paulo. 1980.

2. Chieff PP. Siqueira JGV. Paschoalotti MA. Estudo sobre o metodo de eclosào de miracidios para o dia- gnóstico da esquistossomose mansônica, em inquéritos epidemiológicos. Revista Brasileira de Malariologia e Doenças Tropicais 30:65-75, 1978.

3. Dixon WP, Massey Jr FJ. Introduction to statistical analysis. 2nd. edition, McGraw-Hill, Tokyo, 1957.

4. Kassim O, Gilbertson DE. Hatching of Schistosoma mansoni eggs and observations on motility of miracidia. The Journal of Parasitology 62:715-720, 1976.

5. Mason PR; Fripp PJ. Analysis of the movements of Schistosoma mansoni miracidia using dark-ground photography. The Journal of Parasitology 62:721-729 1976.

6. Pitchford RJ, Visser PS. Some observations on the hatching pattern of Schistosoma mansoni eggs. Annals, of Tropical Medicine and Parasitology 66:399-407, 1972.

7. Siegel S. Estatistica nào-parametrica para as ciências do comportamento. McGraw-Hill. Sào Paulo, 1975.

8. Siqueira JGV, Reys AMO, Ormindo R, Azevedo AMV. Eclosão de miracidios como método de diagnóstico e de avaliação terapèutica na esquistossomose mansónica. Revista Brasileira de Malariologia e Doenças Tropicais 33:86-95, 1981.

9. Souza CP. Rodrigues MS, Azevedo MLL, Araújo N. Estudo comparativo de duas técnicas para obtençào de miracidios de Schistosoma mansoni em fezes humanas. Revista do Instituto de Medicina Tropical de São Paulo 23:251-255, 1981

10. Suzuki N. Color atlas of human helminth eggs. 3rd. edition, JAPC \& JOICFP, Tokyo, 1981.

11. Zicker F, Katz N, Wolf J. Avaliação do teste de eclosão de miracidios na esquistossomose mansónia. Revista do Instituto de Medicina Tropical de Sào Paulo 19:202207, 1977. 